\title{
SÍNDROME DE GOLDENHAR: UMA ABORDAGEM FONOAUDIOLÓGICA
}

\section{Goldenhar Syndrome: speech-language approach}

\author{
Angela Ruviaro Busanello (1), Ana Maria Toniolo da Silva (2), Mara Keli Christmann ${ }^{(3)}$, \\ Marcele Machado Finamor (4), Marília Trevisan Sonego ${ }^{(5)}$, Rafaeli Alves Barcellos ${ }^{(6)}$, \\ Tainara Milbradt Weich ${ }^{(7)}$, Talita Marin Scherer ${ }^{(8)}$
}

\section{RESUMO}

Tema: o objetivo deste trabalho foi relatar o caso de uma criança portadora de Síndrome de Goldenhar em tratamento fonoaudiológico em uma clínica escola. Procedimentos: foram coletados dados do prontuário do paciente, mediante assinatura do Termo de Consentimento Livre e Esclarecido e aprovação no Comitê de Ética da instituição, durante aproximadamente 11 meses de fonoterapia. Trata-se de uma criança de 5 anos e 11 meses de idade, do gênero masculino, apresentando ao nascimento má formação na orelha e no côndilo mandibular direitos, bem como espinha bífida. Para descrição do caso, antes e após o período de intervenção, o paciente passou por avaliações fonoaudiológicas como exame auditivo, do sistema estomatongático, medidas antropométricas faciais, exame articulatório, avaliação fonológica e da discriminação auditiva. Resultados: observaram-se alterações como perda auditiva condutiva de grau moderado na orelha direita; alteração na mobilidade de lábios e língua; funções como deglutição, mastigação e respiração atípicas; medidas antropométricas faciais inferiores à normalidade, principalmente nos ramos mandibulares; bem como distúrbios na fala de ordem fonética (distorções) e fonológicas (dessonorizações). A partir de então, estabeleceu-se um planejamento terapêutico, com resultados positivos no período mencionado, principalmente no que se refere à função de mastigação. Conclusão: o paciente apresentou grande variedade de alterações fonoaudiológicas decorrentes do espectro oculoauriculovertebral, que se somatizam na associação com a respiração oral, os hábitos orais deletérios e os fatores ambientais que atuam no caso. Novos estudos mais aprofundados e com maior número de sujeitos são importantes na corroboração destes resultados.

DESCRITORES: Síndrome de Goldenhar; Sistema Estomatognático; Anormalidades Craniofaciais; Audição; Fala

(1) Fonoaudióloga formada pela Universidade Federal de Santa Maria, UFSM; Fonoaudióloga da Unimed Santa Maria; Mestre em Distúrbios da Comunicação Humana pela Universidade Federal de Santa Maria, Rio Grande do Sul, Brasil.

(2) Fonoaudióloga formada pela Universidade Federal de Santa Maria, UFSM; Professora Adjunta da Universidade Federal de Santa Maria, Santa Maria, Rio Grande do Sul, Brasil; Doutora em Ciências dos Distúrbios da Comunicação Humana - Universidade Federal de São Paulo.

(3) Fonoaudióloga formada pela Universidade Federal de Santa Maria, UFSM; Mestranda do Programa de PósGraduação em Distúrbios da Comunicação Humana pela Universidade Federal de Santa Maria, Santa Maria, Rio Grande do Sul, Brasil.

(4) Fonoaudióloga formada pela Universidade Federal de Santa Maria, UFSM; Técnica Administrativa em Educação da Universidade Federal do Pampa (UNIPAMPA), Uruguaiana, Rio Grande do Sul, Brasil.

(5) Fonoaudióloga formada pela Universidade Federal de Santa Maria, UFSM; Santa Maria, Rio Grande do Sul, Brasil

(6) Fonoaudióloga formada pela Universidade Federal de Santa Maria, UFSM; Santa Maria, Rio Grande do Sul, Brasil.

(7) Fonoaudióloga formada pela Universidade Federal de Santa Maria, UFSM; Mestranda do Programa de PósGraduação em Distúrbios da Comunicação Humana pela Universidade Federal de Santa Maria, Santa Maria, Rio Grande do Sul, Brasil.

(8) Fonoaudióloga formada pela Universidade Federal de Santa Maria, UFSM; fonoaudióloga da Escola Antônio Francisco Lisbôa Santa Maria, Rio Grande do Sul, Brasil.

Conflito de interesses: inexistente 


\section{INTRODUÇÃO}

A Síndrome de Goldenhar (SG) faz parte de um quadro clínico complexo dos primeiro e segundo arcos branquiais, o qual é comumente conhecido como espectro oculoauriculovertebral. Caracterizase por assimetria mandibular, má formação auricular, alterações vertebrais, presença adicional de dermóides epibulbares ${ }^{1,2}$ e, em alguns casos, malformações cardíacas ${ }^{3}$.

Apesar da etiologia desta síndrome ainda ser incerta, estudos recentes demonstram que fatores nutricionais e ambientais, como ingestão de drogas e diabetes materna, podem resultar em distúrbios da blastogênese dos arcos. Outros estudos sugerem que distúrbios na crista neural, anomalias de cromossomos e herança autossômica dominante, recessiva e multifatorial também podem causar esta síndrome ${ }^{1,4}$.

A incidência conhecida da síndrome é de, aproximadamente, um para cada cinco a vinte mil nascidos vivos, acometendo comumente o lado direito da face e do corpo'.

As complicações da SG decorrem, sobretudo, da presença de anomalias craniofaciais. As más formações da orelha externa podem ser desde simples alterações no pavilhão auricular até a ausência completa deste e do meato acústico externo. A face apresenta, no conjunto, características visuais peculiares devido à assimetria facial, hipoplasia do ramo mandibular e do côndilo da mandíbula e ao deslocamento do pavilhão auricular ${ }^{1,2,5}$. Dessa forma, esses achados favorecem o aparecimento de alterações nas estruturas e funções do Sistema Estomatognático (SE).

Entre as funções alteradas do SE, uma das mais prejudicadas é a mastigação, uma vez que o ato de morder, triturar e amassar o bolo estará comprometido principalmente devido às alterações mandibulares. Em crianças portadoras desta síndrome, a assimetria facial e corporal pode não ser totalmente percebida, tornando-se mais evidentes após o surto de crescimento ${ }^{6}$.

A deglutição também pode apresentar alterações, principalmente durante a fase oral. Neste caso, a língua pode ser empurrada contra os dentes frontais ou laterais, ou ser projetada entre os dentes superiores e inferiores. O alimento não é levado corretamente até a zona de trituração pela musculatura lingual e mastigatória débeis, resultando em um bolo alimentar mal formado. Ocorre também contração dos músculos mentual e orbicular, na tentativa de ejeção do bolo para a faringe ${ }^{7}$. A deglutição é dita como adaptada quando ocorre em conseqüência de algum outro problema existente, como má oclusão e respiração oral, pois a língua se adapta à forma da cavidade oral ou tipo facial do indivíduo ${ }^{8}$.
Apesar de não haver necessariamente comprometimentos cognitivos e de linguagem associados a este espectro ${ }^{9}$, a deficiência auditiva provocada por ele é um importante fator de risco para o aparecimento de alterações da linguagem e fala, uma vez que o feedback auditivo é fundamental para a aquisição e monitoramento da comunicação oral ${ }^{10}$.

O desvio fonológico caracteriza-se como uma desorganização, inadaptação ou anormalidade do sistema fonológico das crianças em relação ao sistema padrão de sua comunidade lingüística, na ausência de comprometimento orgânico ${ }^{11}$. Já o desvio fonético é caracterizado pela alteração na produção da fala, decorrente de uma alteração orgânica, seja uma simples distorção na produção do fonema, ou resultante de patologias específicas ${ }^{12}$.

Devido às inúmeras alterações que o paciente com o espectro oculoauriculovertebral pode apresentar e a variabilidade das mesmas, a reabilitação destes pacientes deve ser realizada de forma individual, direcionada às limitações específicas de cada indivíduo por meio de abordagem multidisciplinar.

Assim, o objetivo deste trabalho foi relatar o caso de uma criança portadora de SG em tratamento fonoaudiológico em uma clínica escola.

\section{APRESENTAÇÃO DO CASO}

Este relato caracterizou-se por ser do tipo observacional e exploratório. As informações do paciente foram obtidas pela consulta ao prontuário da clínica escola, no qual foram coletados dados referentes à anamnese, avaliações fonoaudiológica e complementares, hipótese diagnóstica e tratamento.

Referiu-se à descrição do caso de uma criança de cinco anos e onze meses de idade, do sexo masculino, em tratamento na clínica escola desde os quatro anos e cinco meses de idade. Com a finalidade de preservar a identidade do paciente, Ihe foi atribuída a inicial M. O mesmo foi encaminhado por um centro de referência de anomalias craniofaciais do país, devido às dificuldades de mastigação e de fala.

Quanto à história clínica de $\mathrm{M}$, sabe-se que não houve intercorrências durante a gestação e o parto. Apresentou, ao nascimento, malformação na orelha direita, coluna (espinha bífida) e mandíbula (malformação de côndilo mandibular). Iniciou a marcha após um ano e meio de idade, e, segundo a mãe, passou pelo balbucio e as primeiras palavras com um ano e seis meses, bem como as frases simples com dois anos. Ainda assim, apresentava trocas na fala, tornando-se ininteligível ao falar muito rápido. Quanto à alimentação, foi utilizada exclusivamente a consistência pastosa até os dois anos, pois segundo a família, M não gostava de mastigar os alimentos, principalmente frutas. Não foi amamentado no seio 
materno, e encontra-se em processo de retirada da mamadeira, fazendo uso desta quatro vezes por semana. Além disso, apresenta como hábito de mordida a onicofagia, sobremaneira quando fica nervoso. No que se refere ao sono, este é agitado, com a boca aberta, ronco e baba. Quanto aos aspectos fisiopatológicos, apresenta rinite alérgica, sinusite e fez cirurgia para retirar hérnia diafragmática aos 18 meses de idade. M permanece em acompanhamento médico semestral para a malformação na orelha e na coluna. A mãe referiu boa audição para o filho, mas relatou que o canal auditivo da orelha direita não era completo. No que se refere à escolaridade, atualmente cursa a préescola com bom desempenho.

Foram realizadas, antes e após a intervenção, as seguintes avaliações fonoaudiológicas, além das avaliações complementares de neurologia e otorrinolaringologia: avaliação do sistema estomatognático, avaliação antropométrica da face, exame articulatório, avaliação fonológica, avaliação do sistema funcional da linguagem, avaliação auditiva e avaliação da discriminação auditiva. Todas estas avaliações foram analisadas de forma descritiva. Ainda assim, trata-se de um relato de caso e por esse motivo levanta inúmeras questões sobre a relação entre a $S G$ e a Fonoaudiologia. A fim de melhor caracterizar e comprovar esta relação sugere-se que novos estudos sejam realizados com maior número de indivíduos. Esse comentário deve vir no final dos resultados!
Esta pesquisa foi permitida pelo Comitê de Ética e Pesquisa (CEP) da instituição de origem, mediante registro de número 0220.0.243.000-08 e houve consentimento livre e esclarecido no momento de ingresso do paciente na clínica escola.

\section{RESULTADOS}

$\mathrm{Na}$ Avaliação do Sistema Estomatognático observaram-se aspecto, sensibilidade, tônus, postura e mobilidade dos órgãos fonoarticulatórios e suas funções. Foram descritos a partir de então, os aspectos do caso que tiveram alterações.

Quanto às estruturas extra-orais, o paciente não conseguiu manter lábios unidos e inflar as bochechas separadamente, bem como não realizou assobio e vibração de lábios. Quanto às estruturas intra-orais, não realizou vibração, alargamento e afilamento de língua, além de apresentar palato profundo.

No que se refere às funções vegetativas, apresentou deglutição adaptada, havendo projeção lingual anterior, contração de mentual, ação labial e presença de sialorréia. A mastigação, inicialmente bastante semelhante ao processo de mascação, foi simétrica, mas com movimentos lentos. A respiração mostrou-se do tipo misto e de modo oronasal.

Para avaliar a morfologia craniofacial foram obtidas quantitativamente as medidas faciais de $\mathrm{M}$ por meio da avaliação antropométrica da face ${ }^{13}$. Os resultados encontrados são mostrados na Tabela 1.

Tabela 1 - Comparação das medidas antropométricas faciais, em milímetros, do caso analisado pré e pós tratamento e dos padrões de normalidade referidos na literatura para população infantil brasileira

\begin{tabular}{lccc}
\hline Medida antropométrica & $\begin{array}{c}\text { Pré } \\
\text { tratamento }\end{array}$ & $\begin{array}{c}\text { Pós } \\
\text { tratamento }\end{array}$ & $\begin{array}{c}\text { Padrão normalidade } \\
\text { mais próximo à idade } \\
\text { do paciente - 7 anos }\end{array}$ \\
\hline Altura do lábio superior & 16,02 & 16,02 & $17,81-19,08$ \\
Altura do lábio inferior & 34,61 & 34,61 & $38,15-40,37$ \\
Altura do filtro & 9,88 & 9,88 & $12,70-13,94$ \\
Altura do terço superior da face & 48,29 & 48,29 & $51,92-55,63$ \\
Altura do terço médio da face & 48,29 & 48,29 & $47,55-49,87$ \\
Altura do terço inferior da face & 50,74 & 50,74 & $57,16-59,80$ \\
Distância canto externo do olho e cheilion D & 57,03 & 57,03 & $60,08-62,41$ \\
Distância canto externo do olho e cheilion E & 57,03 & 57,03 & $59,04-61,56$ \\
Lateralização mandibular D & 7,47 & 7,47 & $*$ \\
Lateralização mandibular E & 7,48 & 7,48 & $*$ \\
Ramo mandibular D & 66,71 & 66,71 & $*$ \\
Ramo mandibular E & 71,67 & 71,67 & $*$ \\
\hline
\end{tabular}

Legenda: * sem referencial na literatura

Fonte: CATTONI, D.M.; FERNANDES, F.D.M. Medidas e proporções faciais em crianças - contribuições para a avaliação miofuncional orofacial. Pró-Fono Rev. Atual. Cient. 16(1)2004. 
O Exame Articulatório e a Avaliação Fonológica da Criança (AFC) foram realizados com o objetivo de obter amostra de fala com todos os sons da língua em todas as posições em que estes ocorrem no português brasileiro. Verificaram-se inúmeras trocas na fala sem padrão específico, tais como: redução de encontros consonantais e dessonorização (/b/ por /p/, /d/ por /t/, /g/ por /k/ e, /Z/ por /S/). Além disso, ocorreram substituição de /k/ por /t/ e /g/; de /m/ por/b/; // por /n/; omissão de $/ p /$ e apagamento de $/ \eta /$ e de $/ \mathrm{s} /$ em coda medial. Pode-se observar também que há imprecisão articulatória importante, causando distorção na produção da maioria dos fonemas e alteração na nasalidade da voz (esta a ser investigada).

$\mathrm{Na}$ Avaliação do Sistema Funcional da Linguagem foram observadas noções quanto ao esquema corporal, à função temporal, à função espacial e às noções de tamanho e quantidade, todas condizentes com a idade do paciente.

No teste de Weepman, utilizado para avaliação da discriminação auditiva, o paciente apresentou 17 erros em " $x$ " e um erro em " $y$ ", mostrando resultados insatisfatórios para a idade, além de refletir a perda auditiva e a falta de motivação durante a aplicação do teste. É considerado " $x$ " a soma dos erros que a criança disse "igual" para pares de vocábulos diferentes. E "y" a soma dos erros que a criança disse "diferente" para pares de vocábulos iguais. Todos os testes que mostram um escore " $x$ " maior que 15 ou um escore "y" maior que três devem ser invalidados, uma vez que sugerem ter existido algum problema de audição, pouca motivação para o teste ou ainda falta de entendimento das instruções. O escore "y" é utilizado apenas para julgar a validade do teste. Para a idade de cinco anos é considerado insatisfatório um resultado de " $x$ " com mais de seis erros ${ }^{14}$.

A avaliação otorrinolaringológica evidenciou microtia do pavilhão auricular da orelha direita e orelha esquerda sem particularidades. Na avaliação audiológica, os resultados encontrados foram de perda auditiva condutiva de grau moderado na orelha direita e audição normal na orelha esquerda.

Durante a avaliação neurológica, os pais do paciente referiram, pela primeira vez, terem realizado avaliação com equipe multidisciplinar, incluindo avaliação genética geral. Inicialmente $M$ tinha a hipótese da Síndrome de Treacher Collins, porém houve confirmação do diagnóstico de Goldenhar. Esta avaliação indicou aparente normalidade neurológica e neuroevolutiva, apesar do quadro sindrômico manifestado por dismorfias craniofaciais, vertebrais e de órgãos internos.

Após as avaliações realizadas concluiu-se a seguinte hipótese diagnóstica para M: Desvio fonético-fonológico por Perda Auditiva Condutiva de Grau Moderado na orelha Direita e por alterações do Sistema Estomatognático decorrentes de Hábitos Orais Prolongados, Respiração Oral e Malformações Congênitas no Pavilhão Auricular e na Mandíbula de causa sindrômica (espectro oculoauriculovertebral).

Dessa forma, o planejamento terapêutico teve como objetivos gerais adequar os padrões de fala, bem como estruturas e funções do sistema estomatognático e conscientizar o paciente e a família sobre a importância do tratamento fonoaudiológico.

Como objetivos específicos estabeleceram-se:

- Adequar os padrões de fala, trabalhar a discriminação dos fonemas, principalmente os não adquiridos, pois M possuía trocas assistemáticas, sendo os fonemas plosivos, fricativos e líquidos não-laterais os que apresentavam maior dificuldade, além de promover a automatização dos pontos e modos articulatórios corretos.

- Adequar mobilidade de lábios, língua e bochechas; postura de língua; posicionamento e tônus labial; projeção lingual; contração de mentual e ação labial durante a deglutição.

- Realizar treino mastigatório, automatizar os padrões corretos de deglutição; e integrar as novas atividades musculares aos padrões funcionais, utilizando o que o paciente aprendeu durante o desenvolvimento dos novos padrões na deglutição de líquidos e alimentos.

- Esclarecer a família a respeito da importância, dos objetivos e das vantagens do tratamento fonoaudiológico, bem como da assiduidade e cooperação, para que houvesse um efetivo progresso terapêutico.

No período analisado foram realizadas 43 sessões de fonoterapia, totalizando, aproximadamente, 11 meses de encontros semanais, sendo utilizados exercícios isotônicos e isométricos (de forma lúdica) para língua, lábios e bochechas. Foram realizados exercícios de terapia miofuncional oral baseados em Hanson e Barret ${ }^{7}$ para deglutição e exercícios de treino mastigatório de diferentes consistências como goma de mascar envolta em gaze e maçã. Além de estímulos proprioceptivos, auditivos, táteis e visuais para trabalhar os padrões de fala, foram utilizados jogos e brinquedos para promover a imitação do fonema-alvo (/r/), bem como bombardeio auditivo, na orelha esquerda, no início e no final de cada sessão. Ao término de cada atendimento, foram dadas orientações à família.

Ao final deste período, o paciente foi reavaliado fonoaudiologicamente nos mesmos parâmetros iniciais, demonstrando as seguintes evoluções, conforme mostra a Figura 1: 


\begin{tabular}{|l|}
\hline Mastigação \\
\hline Desenvolvimento efetivo da função mastigatória. Apesar de haver o início da instalação da mastigação \\
assimétrica à direita, os avanços são evidentes, pois antes do tratamento fonoaudiológico M só ingeria \\
alimentos liquidificados e pastosos, e agora já está inserindo a consistência sólida em sua alimentação, \\
como maçã e carne. \\
\hline Deglutição \\
\hline Diminuição da projeção lingual durante a deglutição e a redução da sialorréia. \\
\hline Hábitos Orais Deletérios \\
\hline Alternância entre o uso do copo e da mamadeira com a ajuda dos pais \\
\hline Fala \\
\hline $\begin{array}{l}\text { Discreta evolução nas líquidas não-laterais, manifestando produções esporádicas de /r/ retroflexo. } \\
\text { Acredita-se que este progresso tenha ocorrido graças ao estímulo com o som alvo /r/, após inúmeras } \\
\text { tentativas com fonemas fricativos e que não foram bem percebidos e aceitos. }\end{array}$ \\
\hline
\end{tabular}

Figura 1 - Descrição dos parâmetros fonoaudiológicos que apresentaram evolução após período de intervenção fonoaudiológica

A fim de melhor caracterizar e comprovar a existência de determinadas alterações fonoaudiológicas na SG, sugere-se que novos estudos sejam realizados com maior número de indivíduos.

\section{DISCUSSÃO}

A SG é uma constelação heterogênea de malformações, envolvendo classicamente a face, os olhos e as orelhas. O espectro oculoauriculovertebral possui etiologia incerta e afeta predominantemente o lado direito da face e do corpo ${ }^{1,5}$. Essas variáveis condizem com o caso referido, já que $M$ apresentou microtia na orelha direita e hipoplasia do ramo mandibular direito. Além disso, o paciente apresentou ao nascer espinha bífida, uma das anomalias vertebrais encontradas nesta síndrome ${ }^{1}$.

A assimetria facial provocada pela SG pode ocasionar alterações das funções neurovegetativas, como a mastigação e a deglutição². A mastigação é a mais importante função do sistema estomatognático, dependente da mobilidade mandibular, da atividade coordenada da musculatura, dos contatos oclusais e do número de dentes além, é claro, das características do alimento consumido. Essa função envolve uma seqüência precisa de movimentos mandibulares de abertura e fechamento, movimentos dos lábios e bochechas, além da ação complexa da língua ${ }^{15}$.

Assim, salienta-se que a hipoplasia encontrada no paciente relaciona-se estreitamente com sua mastigação assimétrica, pois este utiliza preferencialmente o lado direito nesta função. Durante os dois primeiros anos de vida $M$ foi alimentado apenas com consistência pastosa, contribuindo negativamente para o desenvolvimento das estruturas do $\mathrm{SE}^{2}$. Logo, o padrão mastigatório, somado à consistência alimentar utilizada, podem ter intensificado as alterações neste sistema. Entretanto, a terapia fonoaudiológica proporcionou ao paciente a realização de movimentos rotatórios durante a mastigação, bem como a introdução gradativa alimentos de consistência sólida, favorecendo o desenvolvimento musculoesquelético das hemifaces de forma simétrica.

$\mathrm{Na}$ literatura ${ }^{16}$, há referência de um paciente com SG que, além das manifestações sindrômicas, apresentou deglutição com interposição lingual, anteriorização de cabeça e resíduos alimentares no vestíbulo, mesmo após várias deglutições. Sendo condizente com o caso estudado, M possui deglutição adaptada havendo dois agravantes para o desenvolvimento da mesma, a assimetria facial devido à hipoplasia mandibular e a respiração oronasal.

O indivíduo que substitui o padrão correto de respiração nasal por um padrão inadequado oral ou oronasal é chamado de respirador oral. Durante a fase de crescimento do indivíduo, a persistência da respiração oral pode provocar alterações oclusais, craniofaciais, dos órgãos fonoarticulatórios e das funções estomatognáticas, entre outras. Assim sendo, pode-se dizer que a respiração oral é uma adaptação que influencia negativamente no crescimento e desenvolvimento do esqueleto craniofacial, principalmente no que diz respeito à forma maxilar, mandibular e altura facial ${ }^{17,18}$. A respiração oral quando associada à hipoplasia do ramo mandibular potencializa as alterações no crescimento e desenvolvimento do sistema estomatognático já que ambas afetam estas estruturas ${ }^{16,17}$. 
Os hábitos orais, quando deletérios, também irão interferir nas funções orofaciais constituindo fatores etiológicos em potencial ao desequilíbrio muscular, podendo comprometer o desenvolvimento da oclusão normal, a morfologia e a função do SE. Os hábitos como sucção de dedos ou chupeta podem ser substituídos por hábitos de mordida (onicofagia) ${ }^{19}$, como ocorreu com o paciente. Embora na literatura não seja clara a relação entre espectro oculoauriculovertebral e hábitos orais deletérios, o paciente apresentou onicofagia e uso de mamadeira por tempo prolongado, que somados às alterações sindrômicas agravam as alterações estomatognáticas.

Ao comparar as medidas antropométricas da face de $\mathrm{M}$ com o padrão de normalidade referido na literatura (padrão de referência mais aproximado é de crianças com sete anos, idade superior ao caso em questão), percebe-se que o mesmo encontra-se abaixo do esperado ${ }^{13,20,21}$. Quando se remete às medidas dos ramos mandibulares estas diferenças ficam mais evidentes, salientando a assimetria, já que há diferença de aproximadamente $5 \mathrm{~mm}$ entre os ramos esquerdo e direito da mandíbula.

Sabe-se que entre os cinco e 18 anos de idade ocorre aproximadamente $50 \%$ do crescimento mandibular ${ }^{6}$ e, desse modo, as assimetrias faciais encontradas no paciente poderão tornar-se significativamente maiores. Por esse motivo, a retirada dos hábitos orais e a adequação da respiração e deglutição, objetivando a minimização da sua influencia neste período de desenvolvimento do SE, são fundamentais.

Ainda assim, observa-se que o fato da intervenção fonoaudiológica não ter modificado as medidas antropométricas, não impediu a melhora das funções estomatognáticas, que evoluíram consideravelmente após o tratamento, conforme relatado acima.

Outra alteração encontrada no espectro oculoauriculovertebral é a microtia, ou seja, orelha externa formada anormalmente ou ausente. A microtia unilateral, cerca de seis vezes mais freqüente do que a bilateral, é mais comum em homens do que em mulheres e encontrada predominantemente na orelha direita. $O$ uso de cabelos longos oculta facilmente a deformidade da orelha externa ${ }^{22}$. Os pais do paciente em questão, de fato, mantinham o cabelo do menino comprido com a finalidade de ocultar a má formação da orelha. No caso de $\mathrm{M}$, a obstrução mecânica à condução do som, devido à microtia, levou a perda auditiva condutiva unilateral de grau moderado.
Devido ao fato do desenvolvimento lingüístico depender, em grande parte, do canal sensorial auditivo, uma redução ou eliminação deste canal reduz drasticamente a capacidade de aprender a fala e a linguagem ${ }^{23}$. A perda auditiva caracterizase pela redução da audição em qualquer grau que reduza a inteligibilidade da mensagem falada para a interpretação apurada ou para a aprendizagem. Qualquer tipo de perda auditiva pode comprometer a linguagem, o aprendizado, o desenvolvimento cognitivo e a inclusão social da criança ${ }^{24}$.

Os efeitos da perda auditiva unilateral são menores do que os causados pela perda bilateral, porém, também podem ocasionar problemas. Em presença de ruído ambiental, as crianças com perda unilateral encontram maiores dificuldades que as ouvintes normais para compreender a fala, mesmo quando a orelha melhor está posicionada em direção à fonte sonora, do mesmo modo que sua localização espacial ${ }^{24}$.

Nos casos de perda auditiva de grau moderado, o paciente apresenta dificuldade em ouvir a maioria dos sons da fala durante a conversação e pode apresentar problemas de articulação, como omissões, substituições e distorções na fala ${ }^{24}$. Em outro estudo ${ }^{25}$, observou-se que crianças com SG foram capazes de produzir todas as vogais e consoantes de sua língua materna, e os erros apresentados por elas foram, principalmente, ensurdecimento, distorções, omissão da consoante final e do encontro consonantal. Estes achados são similares ao caso de $\mathrm{M}$, sendo que o mesmo apresentou, entre outras alterações, a dessonorização de forma predominante.

A aquisição e organização dos componentes fonológicos da linguagem estão relacionadas às capacidades internas do ser humano, como a maturação do sistema nervoso central, e também com seu ambiente, que deve ser rico em estímulos e possibilitar diversas experiências lingüísticas $^{16}$. Concordando com a literatura, é possível observar que o ambiente familiar de superproteção, e provável negação, em que o paciente encontravase, pode ter limitado seu desenvolvimento lingüístico, emocional e social. Ao ocultar as informações sobre os comprometimentos que envolvem este espectro, os pais restringiram, de certa forma, a oferta de estímulos nos períodos adequados, principalmente no que se refere à alimentação e à comunicação.

Além da questão fonológica, o menino apresenta distorção na produção dos fonemas, o que vai ao encontro de outros estudos ${ }^{26,27}$ que ao analisar pacientes com SG constataram a presença de alterações articulatórias, apesar de conseguirem produzir todos os fonemas. Distorções como estas 
podem ocorrem em virtude da dificuldade em adequar os pontos articulatórios imposta pelas deformidades craniofaciais.

Desse modo, sugere-se a continuidade do acompanhamento fonoaudiológico com avaliações periódicas dos órgãos fonoarticulatórios e medidas antropométricas visando monitorar o desenvolvimento das estruturas e funções do SE, bem como avaliação da consciência fonológica. Propõem-se novo encaminhamento para o médico otorrinolaringologista com a finalidade de investigar possível má formação nasal, devido a hiponasalidade observada.

\section{CONCLUSÃO}

A partir da análise crítica deste relato e sua comparação com a bibliografia consultada, pode-se concluir que o paciente apresenta alterações fonoaudiológicas nas áreas de motricidade orofacial, audição e fala decorrentes do espectro oculoauriculovertebral. Tais alterações associadas à respiração oral, hábitos orais deletérios e fatores ambientais, estão influenciando negativamente no desenvolvimento lingüístico, das estruturas faciais e suas respectivas funções, agravando o quadro sindrômico.

\section{ABSTRACT}

Background: the purpose of this paper is to tell the case of a child with Goldenhar Syndrome in speechlanguage treatment at a clinical school. Procedures: data were collected from the patient's records, through signature of the Free and Clarified Consent Term and approval in the Ethics Committee of the institution, during approximately 11 months of treatment. This case relates a child with 5 years and 11 months old, male gender, with a birth malformation at the right ear and mandibular condyle, as well as spina bifida. For this description, the patient passed through evaluations of hearing, stomatognathic system, face anthropometric measures, articulation examination, phonological assessment and hearing discrimination, before and after intervention. Results: we noted changes as for conductive hearing loss in a moderate degree at the right ear; modifications in the mobility of lips and tongue; alterations in deglutition, mastication and respiration; reduced face anthropometric measures, mainly at the mandibular ramus; as well as the articulatory (distortions) and the phonological disorders (devoicing). From these data, we set out a therapeutic planning, with positive results in the mentioned period, mainly as for the mastication function. Conclusion: the patient showed several speechlanguage alterations arising from the oculoauriculovertebral spectrum, that is being increased in the association with Mouth Breathing, the deleterious oral habits and the environmental factors of the patient. New studies with more details and higher number of subjects are important to corroborate such results.

KEYWORDS: Goldenhar Syndrome; Stomatognathic System; Craniofacial Abnormalities; Hearing; Speech

\section{REFERÊNCIAS}

1. Paixão MP, Miot HA. Você conhece esta síndrome? An Bras Dermatol. 2007; 83(2): 273-6.

2. Lima RMF, Freire OCB, Filho JLN, Stampford $S$, Cunha DA, Silva HJ. Padrão Mastigatório em crianças de 5 a 7 anos: suas relação com crescimento craniofacial e hábitos alimentares. Rev. CEFAC. 2006; 8(2): 205-15.

3. Rosa RFM, Dall'agnol L, Zen PRG, Pereira VLB, Graziadio C, Paskulin GA. Oculo-auriculo-vertebral spectrum and cardiac malformations. Rev Assoc Med Bras. 2010; 56(1): 62-6.

4. Hartsfield J. Review of the etiologic heterogeneity of the óculo-auriculo-vertebral spectrum (Hemifacial
Microsomia). Orthod Craniofac Res. 2007; 10(3):121-8.

5. Neto FXP, Silva DL, Franco KMS, Nascimento LF, Neville T, Vasconcelos VF. Síndrome de Goldenhar - Um alerta para o Otorrinolaringologista. Arq Int Otorrinolaringol. 2007; 11(2): 196-201.

6. Farkas LG, Posnick JC, Hreczko TM. Growth patterns of the face: a morphometric study. Cleft Palate-craniofacial Journal. 1992; 29(4): 308-15.

7. Hanson ML, Barret RH. Fundamentos da Miologia Orofacial. Rio de Janeiro: Enelivros, 1988.

8. Marchesan IQ. Deglutição - Diagnóstico e Possibilidades Terapêuticas. In: Marchesan IQ. Fundamentos em Fonoaudiologia - Aspectos Clínicos da Motricidade Oral. $2^{\circ}$ ed. Rio de Janeiro: Guanabara Koogan, 2005. p. 51-8. 
9. Silva RCL, Alves FFS, Gonzaga Netto SS, Silva $\mathrm{CM}$. As alterações fonoaudiológicas na síndrome de Goldenhar: relato de caso. Rev Soc Bras Fonoaudiol. 2008; 13(3): 290-5.

10. Mahore A, Dange N, Nama S, Goel A. Facioauriculo-vertebro-cephalic spectrum of Goldenhar syndrome. Neurol Índia. 2010;58(1):141-4.

11. Mota HB, Keske-Soares M, Bagetti T, Ceron MI, Melo Filha MGC. Comparative analyses of the effectiveness of three different phonological

therapy models. Pró-Fono Rev Atual Cient. 2007;19(1):67-74.

12. Marchesan IQ. Alterações de fala de origem musculoesquelética. In: Ferreira LP, Befi-Lopes

DM, Limongi SCO. Tratado de Fonoaudiologia. São Paulo: Roca, 2004. 292-303.

13. Cattoni DM, Fernandes FDM. Medidas e proporções faciais em crianças - contribuições para a avaliação miofuncional orofacial. Pró-Fono Rev Atual Cient. 2004; 16(1): 7-18.

14. Rodrigues E. Discriminação Auditiva (Normas para Avaliação de Crianças de 5 a 9 anos). São Paulo: Cortez, 1981.

15. Rodrigues KF, Leftèvre AP, Mott LB, Tugumia $D$, Pena PL. Análise comparativa entre o lado de predominância mastigatória e medidas da mandíbula por meio do paquímetro. Rev CEFAC. 2003; 5(4): 347-51.

16. Lima FT, Araújo CB, Sousa EC, Chiari BM. Alterações fonoaudiológicas presentes em um caso de síndrome de Goldenhar. Rev Soc Bras Fonoaudiol. 2007; 12(2): 141-5.

17. Marchesan IQ. Avaliação e terapia dos problemas da respiração. In: Marchesan IQ. Fundamentos em Fonoaudiologia - Aspectos Clínicos da Motricidade Oral. $2^{\circ}$ ed. Rio de Janeiro: Guanabara Koogan, 2005. p. 23-36.
18. Bianchini AP, Guedes ZCF, Vieira MM. Estudo da relação entre respiração oral e o tipo facial. Rev Bras Otorrinolaringol. 2007; 73(4): 500-5.

19. Maciel CTV, Barbosa MH, Toldo CA, Faza FCB, Chiappetta ALML. Disfunções orofaciais nos pacientes em tratamento ortodôntico. Rev. CEFAC. 2006; 8(4): 456-66.

20. Cattoni DM, Fernandes FDM. Medidas antropométricas orofaciais de crianças paulistanas e norte-americanas - estudo comparativo. Pró-Fono Rev Atual Cient. 2009;21(1):25-30.

21. Daenecke S, Bianchini EMG, Silva APBV. Medidas antropométricas de comprimento de lábio superior e filtro. Pró-Fono Rev Atual Cient. 2006; 8(3): 249-58.

22. Northern JL, Downs MP. Audição na Infância. 5a ed. Rio de Janeiro: Guanabara Koogan, 2005.

23. Bess FH, Humes LE. Fundamentos de audiologia. 2a ed. Porto Alegre: Artmed, 1998.

24. Viera ABC, Macedo LR, Gonçalves DU. O diagnóstico da perda auditiva na infância. Pediatrias. 2007; 29(1): 43-9.

25. Van Lierde KM, Van Cauwenberge $P$, Stevens I, Dhooge, I. Language, articulation, voice and resonance characteristics in 4 children with Goldenhar syndrome: a pilot study. Folia Phoniatr Logop. 2004; 56(3): 131-43.

26. Belenchia P, Mccardle P. Goldenhar's syndrome: A case study. J Commun Disord. 1985; 18(5): 383-92.

27. D'Antonio LL, Rice RD Jr, Fink SC. Evaluation of pharyngeal and laryngeal structure and function in patients with oculo-auriculo-vertebral spectrum. Cleft Palate Craniof J. 1998; 35(4):333-41.
http://dx.doi.org/10.1590/S1516-18462011005000035

RECEBIDO EM: 03/08/2010

ACEITO EM: 11/01/2011

Endereço para correspondência:

Angela Ruviaro Busanello

Rua General Neto, 484

Santa Maria - Rio Grande do Sul

CEP: 97050 - 240

E-mail: angelafonoaudiologia@yahoo.com.br 